\title{
Planets in Other Universes: Habitability constraints on density fluctuations and galactic structure
}

\author{
Fred C. Adams, ${ }^{a, b}$ Katherine R. Coppess, ${ }^{a}$ Anthony M. Bloch ${ }^{c}$ \\ ${ }^{a}$ Physics Department, University of Michigan, Ann Arbor, MI 48109 \\ ${ }^{b}$ Astronomy Department, University of Michigan, Ann Arbor, MI 48109 \\ ${ }^{c}$ Mathematics Department, University of Michigan, Ann Arbor, MI 48109 \\ E-mail: fca@umich.edu, kcoppess@umich.edu, abloch@umich.edu
}

\begin{abstract}
Motivated by the possibility that different versions of the laws of physics could be realized within other universes, this paper delineates the galactic structure parameters that allow for habitable planets and revisits constraints on the amplitude $Q$ of the primordial density fluctuations. Previous work indicates that large values of $Q$ lead to galaxies so dense that planetary orbits cannot survive long enough for life to develop. Small values of $Q$ lead to delayed star formation, loosely bound galaxies, and compromised heavy element retention. This work generalizes previous treatments in the following directions: [A] We consider models for the internal structure of the galaxies, including a range of stellar densities, and find the fraction of the resulting galactic real estate that allows for stable, long-lived planetary orbits. [B] For high velocity encounters, we perform a large ensemble of numerical simulations to estimate cross sections for the disruption of planetary orbits due to interactions with passing stars. [C] We consider the background radiation fields produced by the galaxies: If a galaxy is too compact, the night sky seen from a potentially habitable planet can provide more power than the host star. [D] One consequence of intense galactic background radiation fields is that some portion of the galaxy, denoted as the Galactic Habitable Zone, will provide the right flux levels to support habitable planets for essentially any planetary orbit including freely floating bodies (but excluding close-in planets). As the value of $Q$ increases, the fraction of stars in a galaxy that allow for (traditional) habitable planets decreases due to both orbital disruption and the intense background radiation. However, the outer parts of the galaxy always allow for habitable planets, so that the value of $Q$ does not have a well-defined upper limit (due to scattering or radiation constraints). Moreover, some Galactic Habitable Zones are large enough to support more potentially habitable planets than the galaxies found in our universe. These results suggest that the possibilities for habitability in other universes are somewhat more favorable and far more diverse than previously imagined.
\end{abstract}




\section{Contents}

1 Introduction 1

2 Galaxy Formation and Galactic Structure $\quad 3$

2.1 Formation of Galaxies 3

2.2 Structure of Galaxies 5

$\begin{array}{lll}3 & \text { Scattering Constraints } & 7\end{array}$

3.1 Interaction Cross Sections 8

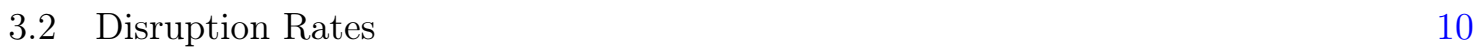

$\begin{array}{lll}3.3 & \text { Survival Fractions for Scattering } & 11\end{array}$

4 Radiation Constraints $\quad 12$

4.1 Radiation Fields within Galaxies 13

$\begin{array}{lll}4.2 & \text { Survival Fractions for Radiation } & 14\end{array}$

4.3 Allowed Regions of Galactic Parameter Space 15

5 Galactic Habitable Zones $\quad 17$

5.1 Extent of the Galactic Habitable Zone 18

$\begin{array}{lll}5.2 & \text { Properties and Consequences } & 20\end{array}$

6 Conclusion 22

6.1 Summary of Results 22

6.2 Discussion 23

\section{Introduction}

One of the most important cosmological parameters is the amplitude $Q$ of the primordial density fluctuations. In our universe, this quantity is small, $Q \sim 10^{-5}$, as measured by the corresponding temperature fluctuations in the cosmic microwave background [1-3]. The parameter $Q$ sets the initial conditions for the growth of structure in standard cosmological models, where dark matter provides most of the matter available for structure formation and dark energy provides most of the energy density of the universe [2].

A key question is why the amplitude $Q$ has this particular small value. The temperature fluctuations observed in the cosmic background radiation are thought to arise from quantum fluctuations produced during the inflationary epoch [4] in the early universe. However, the observed smallness of the parameter $Q$ requires a corresponding small parameter in the scalar field potential of inflationary models $[5,6]$. Other choices of the scalar field potential generally tend to produce larger fluctuations and hence larger values of $Q$ (see the discussion of [7]). Given the possible existence of other universes, perhaps as part of a multiverse, it is reasonable to expect that these other regions of space-time sample a distribution of values for $Q$. Previous authors $[8,9]$ have considered this possibility and placed bounds on the values of $Q$ that allow for structure formation with favorable properties. In general, if the fluctuation amplitude $Q$ is too small, then cosmological structure has difficulty forming, and the resulting objects could be too rarified to cool and form stars $[8,10,11]$; in addition, they could be too loosely 
bound to retain the heavy elements produced by stars. On the other hand, if $Q$ is too large, then galaxies are much denser than those of our universe; they can be too dense to allow for planetary orbits to remain unperturbed over the long time scales necessary for biological evolution to run its course.

The consideration of alternate universes - in this case with different values of $Q$ and hence different types of galactic structures - is counterfactual in nature. The laws of physics are described by a collection of fundamental constants, but we currently have no definitive explanation for why these constants have their measured values. Similarly, the standard picture of cosmology requires a corresponding set of cosmological parameters, with specific values required to match observations, but we have no a priori theory to predict these values. One possible - but only partial - explanation is that our universe is part of a larger space-time structure, often called the multiverse [12-14], where each separate universe represents a different realization of the laws of physics within the ensemble of possible universes. More specifically, each separate region of space-time can have its own values for both the fundamental constants of physics and for the cosmological parameters. Our universe - our region of space-time - thus has one particular realization of these quantities, drawn from some underlying distribution of parameters.

This possibility motivates the present paper: If the multiverse concept can provide even a partial explanation for why our universe has its observed properties, then we must first understand what range of parameter space allows for a universe in which life (more or less as we know it) can develop [9, 15-18]. Note that in order to determine the probability of life arising in a universe, we must not only determine the parameters that allow for life but also the probability of realizing those parameter values [19]; thus far, we have little understanding of the underlying probability distributions. As a result, this paper has the modest goal of constraining what range of galactic properties allows for the survival of habitable planets, and using the result to contrain the allowed values of the fluctuation amplitude $Q$.

The question of structure in other universes, including constraints on the primordial fluctuation amplitudes [9], has been considered previously [8, 16, 20]. In general, these prior treatments adopt a global approach, where a large number of constraints are presented and calculations are carried out in an order-of-magnitude fashion (see also [21]). Building on these previous studies, this paper focuses on the specific issue of how galactic environments can disrupt potentially habitable solar systems. This work explores the problem in greater detail by considering the internal structure of galaxies (including a range of densities), numerical simulations of the cross sections for orbital disruption, and the radiation fields provided by the background galaxy. This paper thus complements previous studies.

Within our Galaxy, taking inventory of habitable planets has now become observationally possible. The first planet with an Earth-like mass that resides in the habitable zone of a mainsequence star has recently been detected [22]. Moreover, projections suggest that the fraction of all Sun-like stars that have Earth-like planets in habitable orbits is large, of order 10 percent [23]. This fraction of potentially habitable planets could be even higher for smaller stars, which are far more numerous. Although long anticipated, a large population of Earthlike planets is rapidly becoming an observational reality.

In universes with different choices of the primordial fluctuation amplitude $Q$, the density of stars in the resulting galaxies varies. For large $Q$, galaxies collapse early, while the background universe is dense; the resulting galaxies are more compact, so that the habitability of Earth-like planets can be compromised in two conceptually different ways: [A] Potentially habitable planets can be scattered out of their solar systems when the stellar density is suf- 
ficiently high. $[\mathrm{B}]$ The planetary surfaces can become too hot due to the background stellar radiation fields. As an order of magnitude estimate, both of these mechanisms start to compromise the survival of habitable planets when galaxies are denser than those of our universe by a factor of $\sim 3 \times 10^{5}$. However, galaxies have internal structure, so that the stellar density varies enormously within such an extended stellar system. As a result, we consider galactic models and find the fraction of the stars within the galaxies that allow for habitable solar systems. This generalization allows some fraction of solar systems to survive, even in galaxies with extreme properties.

The rest of this paper is organized as follows. The current paradigm for cosmological structure formation is briefly outlined in Section 2, where we discuss how the properties of dark matter halos scale with the amplitude $Q$ and then specify models for galactic structure. Section 3 presents results for the disruption of solar systems by scattering encounters, including calculation of interaction cross sections, disruption rates, and the fraction of solar systems that survive. Section 4 presents the analogous results for disruption due to radiation by calculating the background radiation fields of the galaxies and determining the fraction of solar systems that avoid overheating. The background radiation fields of dense galaxies provide regions where freely floating planets have the proper temperature to support liquid water on their surfaces; the extent of these Galactic Habitable Zones is explored in Section 5. Finally, we conclude in Section 6 with a summary of our results and a discussion of their implications.

\section{Galaxy Formation and Galactic Structure}

\subsection{Formation of Galaxies}

In this section, we use the basic cosmological framework for structure formation, as developed for our universe, to determine the types of structure that could form in other universes with alternate values of the fluctuation amplitude $Q$. Since we want to consider cases that are markedly different from our own, we use only the most basic elements of the current theory. To start, we assume that the universe is spatially flat and that the matter content is dominated by collisionless dark matter. In this context, the basic elements of structure formation with varying $Q$ have been outlined previously for universes containing only dark matter [8] and including dark energy [9]. The derivation presented below follows these earlier papers (see $[8,9]$ for further detail).

Density fluctuations begin to grow at the end of the radiation dominated era and become nonlinear after the overdensity reaches a critical value. For the case of a top-hat density perturbation with spherical symmetry, linear perturbation theory [24] predicts that nonlinear collapse ensues at an overdensity of $\sim 1.7$ (see also [25]). At the epoch of matter/radiation equality, the temperature $T_{\mathrm{eq}}$ of the universe is given by

$$
T_{\text {eq }}=\eta m_{P} \frac{\Omega_{M}}{\Omega_{\mathrm{b}}},
$$

where $m_{P}$ is the proton mass, $\eta$ is the baryon-to-photon ratio, $\Omega_{M}$ is the dark matter energy density relative to the critical density, and $\Omega_{\mathrm{b}}$ is the corresponding energy density in baryons $[9,26]$. Note that we are working in units where $c=\hbar=k=1$, so that $G=M_{\mathrm{pl}}^{-2}$ (i.e., $M_{\mathrm{pl}}$ is the Planck mass). Notice also that $T_{\text {eq }}$ is essentially the mass in non-relativistic matter per 
photon. $^{\dagger}$ The age of the universe at equality is then given by

$$
t_{\mathrm{eq}}=\frac{1}{8}\left(\frac{3}{\pi}\right)^{1 / 2} \frac{M_{\mathrm{pl}}}{a_{R}^{1 / 2} T_{\mathrm{eq}}^{2}} \approx \frac{M_{\mathrm{pl}} \Omega_{\mathrm{b}}^{2}}{8 a_{R}^{1 / 2}\left(\eta m_{P} \Omega_{M}\right)^{2}} .
$$

The mass scale of the horizon at the epoch of matter domination plays an important role and is given by

$$
M_{\mathrm{eq}} \approx \frac{1}{64} \frac{M_{\mathrm{pl}}^{3}}{T_{\mathrm{eq}}^{2}} \approx 4 \times 10^{71} \mathrm{GeV} \approx 4 \times 10^{14} M_{\odot},
$$

where we have used $\eta=10^{-9}$ and $\Omega_{M} / \Omega_{\mathrm{b}}=6$, values appropriate for our universe, to obtain the numerical estimates. Fluctuations cannot grow before the epoch of matter domination, so that all mass scales $M<M_{\text {eq }}$ start with roughly equivalent fluctuation amplitudes and grow to become virialized at the later time given by

$$
t_{\mathrm{vir}} \approx t_{\mathrm{eq}} Q^{-3 / 2} f_{\mathrm{vir}}
$$

where the dimensionless parameter $f_{\text {vir }} \lesssim 1$ is a slowly varying function of the halo mass [8]. In actuality, the virialization time (2.4) becomes independent of $M$ only in the limit of small mass scales; by using this approximation, we are thus encapsulating the mass dependence of the virialization time into the parameter $f_{\text {vir }}$. Galaxies with smaller masses collapse to somewhat higher densities because they collapse earlier, when the universe was denser, and the density of the background universe at the time of collapse is a controlling factor in the problem (see Section 2.2 and especially Figure 1 of reference [8] for further discussion).

After a galactic halo structure collapses, it has a characteristic density given by a factor of $f_{\mathrm{c}} \approx 18 \pi^{2}$ times the density of the background universe at time $t_{\mathrm{vir}}$, i.e.,

$$
\rho_{\mathrm{c}}=f_{\mathrm{c}} \rho\left(t_{\mathrm{vir}}\right)=18 \pi^{2} \rho_{\mathrm{eq}}\left(\frac{t_{\mathrm{eq}}}{t_{\mathrm{vir}}}\right)^{2}=18 \pi^{2}\left(2 a_{R} T_{\mathrm{eq}}^{4}\right) Q^{3} f_{\mathrm{vir}}^{-2}
$$

Inserting typical values, we obtain

$$
\rho_{\mathrm{c}} \approx 5.5 \times 10^{-14} \mathrm{~g} \mathrm{~cm}^{-3} Q^{3} f_{\mathrm{vir}}^{-2}=8.2 \times 10^{8} M_{\odot} \mathrm{pc}^{-3} Q^{3} f_{\mathrm{vir}}^{-2} .
$$

For mass scales $M<M_{\mathrm{eq}}$, the characteristic densities of the collapsed structures are roughly comparable and given by equations (2.5) and (2.6). The density increases slowly with decreasing galactic mass due to the factor $f_{\text {vir }}$. Since we are interested in constraints produced by the densest galactic structures, and since galaxies in our universe have masses $M<M_{\text {eq }}$, this paper focuses on this "low-mass" regime.

To obtain numerical values for equation (2.6), we have used the baryon-to-photon ratio $\eta=10^{-9}$ and the mass density ratio $\Omega_{M} / \Omega_{\mathrm{b}}=6$, although these quantities are expected to vary from universe to universe. As a result, one should keep in mind that the characteristic density scales according to the relation $\rho_{\mathrm{c}} \propto Q^{3} T_{\mathrm{eq}}^{4}$. For ease of presentation, the results of this paper are given as constraints on value of $Q$, but the results more generally apply to the composite parameter $Q T_{\mathrm{eq}}^{4 / 3}$. In other words, larger values of the fluctuation amplitude $Q$ can be offset by invoking alternate values of $\left(\eta, \Omega_{M}, \Omega_{\mathrm{b}}\right)$ that lead to smaller values of $T_{\text {eq }}$ (keeping the composite parameter constant). On the other hand, inflationary scenarios tend

\footnotetext{
${ }^{\dagger}$ Finally, note that we ignore the contribution of neutrinos in defining $T_{\text {eq }}$.
} 
to produce a wide range of possible fluctuation amplitudes, often larger than those in our universe, so it makes sense to use $Q$ as the primary variable.

For completeness, we note that for mass scales $M>M_{\text {eq }}$ collapse occurs later, so that these large structures become nonlinear (as density perturbations) at later times given roughly by

$$
t \approx t_{\mathrm{eq}} Q^{-3 / 2} f_{\mathrm{vir}}\left(M / M_{\mathrm{eq}}\right)
$$

Alternatively, the mass scales of these larger structures are a fraction of the horizon mass,

$$
M \sim Q^{3 / 2} M_{\text {hor }} \sim \frac{4 \pi}{3} \rho r_{\text {hor }}^{3},
$$

where $r_{\text {hor }}$ is the horizon scale at the time when the perturbations become nonlinear. The corresponding length scale $r$ is given by

$$
r \sim Q^{1 / 2} r_{\text {hor }} .
$$

These more massive structures have lower characteristic densities by a factor of $\sim\left(M_{\text {eq }} / M\right)^{2}$. As a result, the lower-mass regime considered above produces galaxies that are more disruptive to their constituent solar systems.

The considerations outlined above apply to the initial formation of cosmological structures. As the universe in question evolves, structures on all scales tend to interact and merge, thereby creating a complicated network of interactions. Merging continues until the dark energy component of the universe (if nonzero) dominates and effectively freezes out further structure formation [9, 27-29]. While operative, the merging process tends to produce everlarger structures and acts to redistribute the mass - both the internal structure of individual halos and the mass distribution of the ensemble of halos. In general, merging structures result in more massive structures with lower density. Since we are primarily interested in the densest structures, however, we focus on halos with masses $M \lesssim M_{\text {eq }}$.

\subsection{Structure of Galaxies}

Numerical simulations of cosmological structure formation indicate that dark matter halos of galaxies and clusters approach a nearly universal form. The benchmark study of this convergence [30] showed that the density distribution of the halos assume what is now called the NFW profile, which can be written as

$$
\rho=\frac{\rho_{0}}{\xi(1+\xi)^{2}} \quad \text { where } \quad \xi=\frac{r}{r_{0}}
$$

where $r_{0}$ is the scale length of the system. This simple form for the density distribution cannot continue out to arbitrarily large radii (as the enclosed mass would diverge). However, a number of additional effects act to provide halo edges. At the present cosmological epoch, halos with this density distribution meet up with their neighboring halos, which provide an effective outer boundary. At later times, however, the accelerating cosmic expansion leads to the isolation of halos, which approach an asymptotic form $[28,29]$ with a somewhat steeper density distribution with the form of a Hernquist [31] profile,

$$
\rho=\frac{\rho_{0}}{\xi(1+\xi)^{3}} \quad \text { where } \quad \xi=\frac{r}{r_{0}} .
$$

These same simulations show that a truncation radius develops in above density distribution, where the asymptotic value $r_{T} \approx 4.6 r_{200}$ (see Figure 3 of [28]; see also [32]). 
In this work, we assume that dark matter halos have the form given by equation (2.11). For simplicity, we also assume that the stellar component of the galaxies have the same form, albeit with a more concentrated configuration. In other words, we also use the Hernquist profile to specify the distibution of stars for a model galaxy. We can then calculate the density as a function of radius. At sufficiently large distances, the density will decrease enough so that planets can survive the disruptive effects of both scattering and radiation.

The (crude) theory of structure formation outlined above shows that for a universe with a given value of $Q$, all halos with moderately low mass $M<M_{\mathrm{eq}}$ will virialize at roughly similar times and have densities $\rho_{\mathrm{c}}$ given by equation (2.5) after collapse. Here we make the identification that

$$
\rho_{\mathrm{dm}}=\rho_{\mathrm{c}},
$$

where $\rho_{\mathrm{dm}}=\rho_{0}$ is the density scale appearing in the density distribution (2.11) for the dark matter. For a given total halo mass $M$, the corresponding length scale $r_{\mathrm{dm}}$ for the halo is given by

$$
r_{\mathrm{dm}}=\left(\frac{M}{2 \pi \rho_{\mathrm{c}}}\right)^{1 / 3}=\left(\frac{M}{M_{\mathrm{eq}}}\right)^{1 / 3} \frac{M_{\mathrm{pl}}}{8 \pi T_{\mathrm{eq}}^{2}}\left(\frac{f_{\mathrm{vir}}^{2}}{9 a_{R}}\right)^{1 / 3} Q^{-1} .
$$

With the parameters of the dark matter halo specified, we need to determine the corresponding parameters for the baryonic component. Since gas can dissipate energy, one expects the baryons to collapse further and attain a more centrally concentrated configuration. Because the ratio of the total mass in baryons to that in dark matter is determined by $\Omega_{M} / \Omega_{\mathrm{b}}$, only one additional parameter needs to be specified. We take this parameter to be the ratio $R_{\rho}$ of the density scales of the two components, i.e.,

$$
R_{\rho} \equiv \frac{\rho_{\mathrm{b}}}{\rho_{\mathrm{dm}}} .
$$

Keep in mind that $\rho_{\mathrm{b}}$ (respectively, $\rho_{\mathrm{dm}}$ ) is the density scale appearing in the baryonic (dark matter) density profile, both of which are assumed to have the Hernquist form given by equation (2.11). The scale length $r_{\mathrm{b}}$ for the baryonic component is then given by

$$
r_{\mathrm{b}}=r_{\mathrm{dm}}\left(\frac{\Omega_{\mathrm{b}}}{\Omega_{\mathrm{M}}} \frac{\rho_{\mathrm{dm}}}{\rho_{\mathrm{b}}}\right)^{1 / 3}=r_{\mathrm{dm}}\left(\frac{\Omega_{\mathrm{b}}}{\Omega_{\mathrm{M}} R_{\rho}}\right)^{1 / 3} .
$$

If the star formation rate is uniform across the galaxy, the number density $n_{*}(\xi)$ of stars also has the form

$$
n_{*}=\frac{n_{0}}{\xi(1+\xi)^{3}} \quad \text { where } \quad \xi=\frac{r}{r_{\mathrm{b}}} .
$$

The total number of stars in the (spherical) galaxy is given by

$$
N_{*}=2 \pi r_{\mathrm{b}}^{3} n_{0},
$$

where the stellar density scale $n_{0}$ is given by

$$
n_{0}=\frac{\epsilon_{\mathrm{sf}} \rho_{\mathrm{b}}}{\left\langle M_{*}\right\rangle},
$$

where $\epsilon_{\mathrm{sf}}$ is the star formation efficiency of the galaxy (the fraction of the baryonic mass within the galaxy that has been processed into stars) and $\left\langle M_{*}\right\rangle$ is the mean stellar mass. 
This treatment for the baryonic component makes several assumptions: First we assume that the gas can cool and successfully make stars. In order to cool promptly, on time scales comparable to the age of the universe at the epoch of formation, the gas must be sufficiently dense. In this context, previous work shows that for $Q \gtrsim 3 \times 10^{-5}$, the gas can cool for essentially all mass scales of interest [8]. As a result, the cooling criterion is expected to be satisfied for most galaxies in the large- $Q$ universes considered in this paper.

In our universe, during galaxy formation the gas often falls inward to form disk-like structures, rather than the simple spherical profiles considered here. In addition to providing a useful working model for galactic structure, the quasi-spherical density profiles invoked here are likely to arise for a number of reasons: The characteristic length scales for galactic structure decrease with increasing $Q$. In our galaxy, and most other spirals in our universe, the inner regions (on small length scales) display nearly spherical geometry in the form of galactic bulges. In fact, the Hernquist profile (equation [2.11]), which characterizes the asymptotic form of dark matter halos [28], was originally developed as a model for galactic bulges [31]. This form for the density profile also arises in a wide variety of other cold collapse scenarios (e.g., [33, 34]). If cooling and star formation proceeds rapidly, galaxies are expected to resemble scaled-up bulges, rather than disks. Even if they form initially, disk structures can relax dynamically by scattering stars and adjusting their structure into more rounded configurations [35]. For our galaxy, the disk will relax over a long time scale of order $\sim 10^{19} \mathrm{yr}$ $[36,37]$. For the densest galaxies under consideration, the relaxation time can become shorter than the typical time for habitability (usually taken to be $\sim 1 \mathrm{Gyr}$ ), but will often be longer. However, a particular kind of orbit instability - namely resonant motion perpendicular to the galactic disk - can force stars (or parcels of gas) to leave their orbital planes in even mildly triaxial systems [38]. In many cases, the time scale for this type of instability to alter the phase space of a stellar system is much shorter than the time scale for two-body relaxation [39]. Finally, merging of smaller structures plays an important role in determining the form of bulges and ellipticals in our universe, and mergers could be even more important in the high- $Q$ universes of interest here. This paper thus assumes that the combination of rapid collapse, mergers, orbit instability, and dynamical relaxation leads to rounded galactic structures.

\section{Scattering Constraints}

In this section we estimate the disruption probability for solar systems in the idealized galactic models described above. The rate $\Gamma$ of scattering encounters between a solar system and passing field stars is given by

$$
\Gamma=n_{*} \sigma v,
$$

where $n_{*}$ is the stellar density, $\sigma$ is the interaction cross section, and $v$ is the relative speed between the solar system in question and passing stars.

We start with an order of magnitude estimate: In our Galaxy today [40], the stellar density $n_{*} \approx 0.2 \mathrm{pc}^{-3}$ and the random encounter speed $v \approx 40 \mathrm{~km} \mathrm{~s}^{-1}=40 \mathrm{pc} / \mathrm{Myr}$. At these speeds, the cross section for disrupting the orbit of Earth is approximately $\sigma \approx 50 \mathrm{AU}^{2}$ $=1.25 \times 10^{-9} \mathrm{pc}^{2}[41]$, where disruption corresponds to an increase in eccentricity to values above $e>0.5$ (with this eccentricity, the stellar flux varies by a factor of 9 , nearly an order of magnitude, over the orbit). With these values, the benchmark interaction rate for the disruption of our Solar System becomes

$$
\Gamma=10^{-8} \mathrm{Myr}^{-1}=10^{-5} \mathrm{Gyr}^{-1} .
$$


Although the time scale required for life to develop is not known, 4.6 Gyr is apparently long enough and the value of $t_{\mathrm{c}}=1 \mathrm{Gyr}$ is often used as a standard time scale required for habitability $[42,43]$. Adopting this value, we would have to increase the stellar density by a factor of $\sim 10^{5}$ in order for the interaction rate to be high enough to compromise habitability (keeping other parameters fixed).

\subsection{Interaction Cross Sections}

In this work, we need to calculate the cross sections for disruption of planetary orbits due to interactions between a solar system and passing stars. Previous work has explored these cross sections for the lower speeds appropriate for star forming cluster environments [44, 45] and for field stars in our galaxy [41]. For the dense, highly interactive galaxies of interest in this work, we need the disruption cross sections for higher encounter speeds.

For the sake of definiteness, we focus on the case of a single planet system interacting with a single passing star ${ }^{\dagger}$ and take the system to be an analog of the Earth-Sun system: The host star mass $M_{\text {host }}=1 M_{\odot}$, the planet mass $M_{P}=1 M_{\oplus}$, and the initial planetary orbit is circular $(e=0)$ with semimajor axis $a=1 \mathrm{AU}$. Even in this simplified setting, the interactions must be described by a large number of additional variables, including the mass $M_{*}$ of the passing star, the impact parameter $b$ of the encounter, the relative velocity $v_{r}$ of the two stars at infinite separation, two angles $(\theta, \phi)$ that specify the orientation of the solar system with respect to velocity vector of the incoming star, and the phase angle of the planetary orbit at the start of the interaction. We approach this problem using a Monte Carlo scheme to sample the distributions of all of the aforementioned variables. The stellar masses of the passing stars are drawn from the initial mass function, which is assumed to have the same form as that found in our universe. The angles are drawn randomly such that the passing star is equally likely to approach from any direction and the phase angle of the orbit is uniform-random. The relative velocity is drawn from a Maxwell-Bolzmann type distribution of the form $d f / d v$ $\propto v^{2} \exp \left[-v^{2} / v_{\mathrm{b}}^{2}\right]$. The velocity scale $v_{\mathrm{b}}$ of the distribution characterizes velocity dispersion of the background galaxy, where the mean value $\langle v\rangle=2 v_{\mathrm{b}} / \sqrt{\pi} \approx 1.128 v_{\mathrm{b}}$. Note that the galaxy could, in principle, have additional rotational velocities.

With the variables chosen for a given interaction, the Newtonian equations of motion are integrated numerically using a Bulirsch-Stoer algorithm. Note that the integrations must be carried out to high accuracy: We need to resolve the orbital elements of the planet at the end of the simulation; the planet carries energy of order $M_{P} v_{\mathrm{orb}}^{2}$, whereas the energy of the interaction is much larger, of order $M_{*} v_{\mathrm{b}}^{2}$. The orbital speed $v_{\text {orb }} \sim 30 \mathrm{~km} \mathrm{~s}^{-1}$, but we need to consider relative speeds as large as $v_{\mathrm{b}} \sim 10^{4} \mathrm{~km} \mathrm{~s}^{-1}$; the ratio of these energies is thus $\sim 10^{-10}$, so the numerical scheme must conserve energy at an even greater level of precision. For these simulations, the time step is reduced accordingly so that this criterion is met (see also [41, 44, 45] for further discussion).

For each given velocity dispersion of the background galaxy, we have performed an ensemble of numerical simulations of interactions between single planet solar systems and passing stars. The results are then used to find the cross sections for a given change in the orbital elements of the Earth-analog planet [41, 44, 45]. This paper reports the results from scattering interactions using five choices of the velocity $v_{\mathrm{b}}$ that sets the scale of the velocity distribution; specifically we use $\log _{10}\left(v_{\mathrm{b}} / 1 \mathrm{~km} \mathrm{~s}^{-1}\right)=2,2.5,3,3.5$, and 4 (i.e., the velocity scale $v_{\mathrm{b}}$ is distributed evenly in the logarithm from 100 to $10^{4} \mathrm{~km} \mathrm{~s}^{-1}$ ). The total number

\footnotetext{
${ }^{\dagger}$ For the dense galaxies of interest, binary systems act like two separate single stars because the encounter speeds are much larger than binary orbit speeds [45].
} 


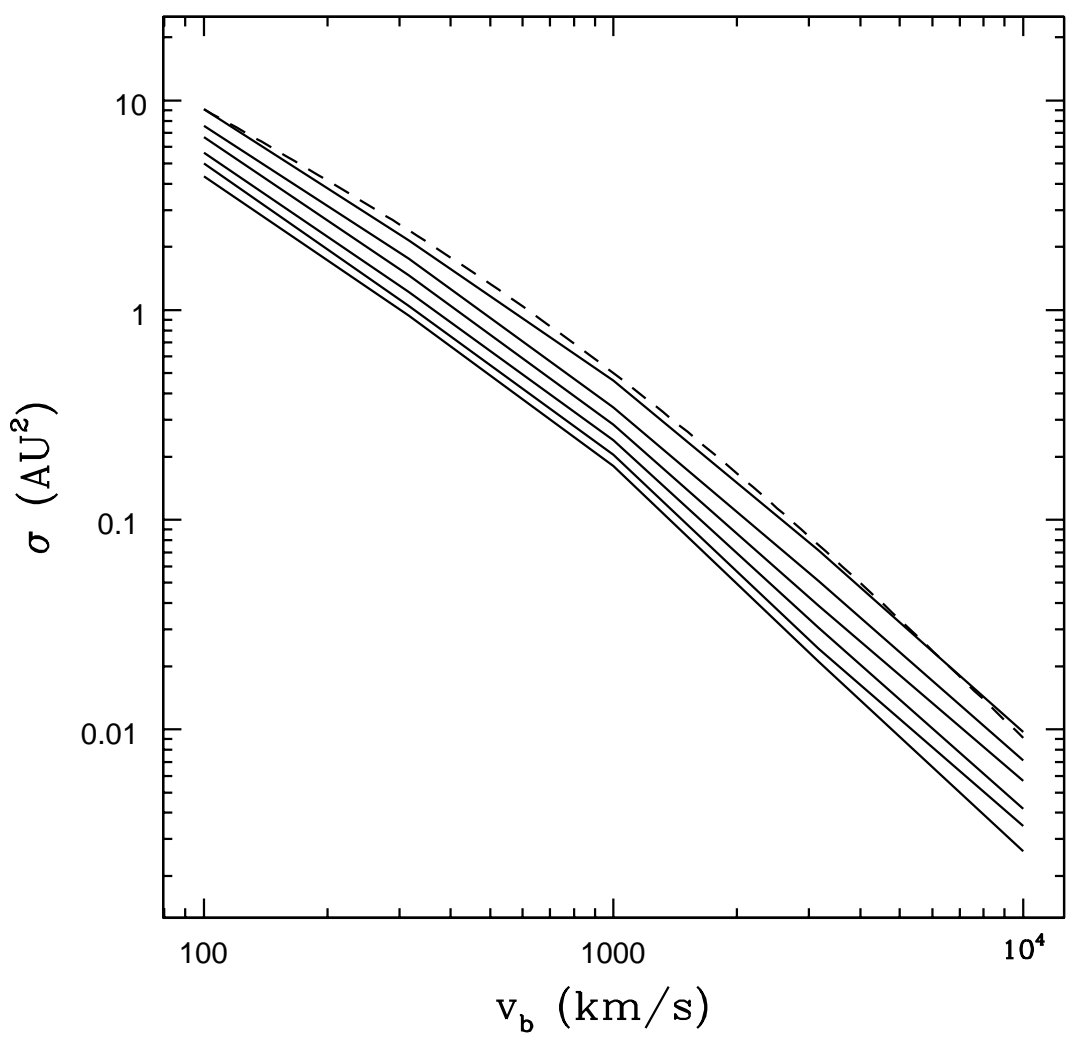

Figure 1. Cross sections for the disruption of a single-planet solar system due to passing stars. Cross sections are shown for increasing the orbital eccentricity to values of $0.5-1.0$, or greater, from top to bottom, where post-encounter values $e \geq 1$ correspond to planetary ejection. The dashed curves shows a simple analytic fit to the cross section for obtaining $e>0.5$ (see text).

of numerical experiments was of order one million, with a greater number of realizations required for larger velocities. With the simulations completed, we then post-process the results to determine the probabilities and the corresponding cross sections for any particular outcome to occur (details of this procedure are given in [41, 44, 45]).

The resulting cross sections are shown in Figure 1 for galactic velocity dispersions in the range $v_{\mathrm{b}}=10^{2}-10^{4} \mathrm{~km} \mathrm{~s}^{-1}$. Even with the large number of realizations, the Monte Carlo sampling errors for these cross sections are about $5 \%$. We also note that these cross sections are in good agreement with extrapolations from previous simulations performed at lower impact speeds $[41,45]$. The figure shows the cross sections for increasing the eccentricity of the planet from its starting value $(e=0)$ to a post-encounter value of $e=0.5-1$, where the $e=1$ final states represent ejections of the planet (including both stripping the planet from its bound orbit and driving the eccentricity to unity so that the planet is accreted by its host star). For purposes of this paper, we consider $e>0.5$ to represent sufficient disruption for compromising planetary habitability and thus adopt the upper curve in Figure 1 as the destruction cross section.

Figure 1 shows that the disruption cross sections are steeply decreasing functions of the velocity dispersion (set by $v_{\mathrm{b}}$ ) of the galaxy. The computed cross section for increasing the eccentricity to $e>0.5$ (the case of interest) is comparable to the geometric cross section of 
the orbit $\left(A=\pi a^{2} \approx 3.1 \mathrm{AU}^{2}\right)$ for velocity dispersion $v \sim v_{0}=1000 \mathrm{~km} \mathrm{~s}^{-1}$. As a result, to a good approximation, the cross section for disruption $\sigma_{\text {dis }}$ can be fit with a simple function of the form

$$
\sigma_{\text {dis }} \approx \frac{\sigma_{0}}{u(1+u)} \quad \text { where } \quad u \equiv \frac{v}{v_{0}}=\frac{v}{1000 \mathrm{~km} \mathrm{~s}^{-1}},
$$

where $\sigma_{0}=1 \mathrm{AU}^{2}$. This expression captures the numerically discovered behavior that the cross section falls more steeply when the velocity dispersion $v \gtrsim v_{0}=1000 \mathrm{~km} \mathrm{~s}^{-1}$ and approaches the form $\sigma \propto v^{-2}$ in the limit $v \rightarrow \infty$. This latter asymptotic form can be derived analytically using the impulse approximation and assuming that the passing star acts on the planet alone (so that the interaction with the host star is negligible). This latter assumption only holds in the limit where the cross section is much smaller than the geometric cross section and hence in the high speed limit.

\subsection{Disruption Rates}

With the interaction cross sections and the structure of the galaxy specified, we consider the disruption rates for solar systems residing at varying locations. Within the context of this galactic model, some portion of the the inner galaxy will always be too dense for solar systems to survive (because $n_{*} \propto 1 / \xi$ ). Similarly, some solar systems can survive in any galaxy provided that the system resides at a sufficiently distant orbit. The goal is thus to determine that fraction of the stellar population that could in princple harbor habitable planets.

The disruption rate for solar systems is given as a function of position within the galaxy by the expression

$$
\Gamma=\frac{n_{0} \sigma v}{\xi(1+\xi)^{3}},
$$

and the corresponding requirement that solar systems survive for a given time $t_{\mathrm{c}}$ takes the form

$$
n_{0} \sigma v t_{\mathrm{c}}<\xi(1+\xi)^{3},
$$

where $\xi=r / r_{\mathrm{b}}$. As outlined above, this paper adopts the characteristic time scale $t_{\mathrm{c}}=1$ Gyr $[42,43]$, which is a resonable estimate for the time required for biological evolution to occur. The reader can scale the results for different choices of this time scale. Using the fitting function for the interaction cross section from equation [3.3], this constraint takes the form

$$
n_{0} \sigma_{0} v_{0} t_{\mathrm{c}}<\xi(1+\xi)^{3}(1+u),
$$

where the scaled speed $u$ is a function of galactic position. To evaluate the speed $u$, we use circular orbits (of the solar system, around the galaxy) in the potential of a dark matter halo with the density distribution of equation (2.11), and find

$$
u=\left(\frac{G M}{r_{\mathrm{dm}} v_{0}^{2}}\right)^{1 / 2} \frac{\left(\xi r_{\mathrm{b}} / r_{\mathrm{dm}}\right)^{1 / 2}}{1+\xi r_{\mathrm{b}} / r_{\mathrm{dm}}} .
$$

The factors of $r_{\mathrm{b}} / r_{\mathrm{dm}}$ arise because the orbital speed is determined by all of the mass in the galaxy, which is dominated by dark matter, whereas the number density of stars (and hence $\xi)$ depends on the baryonic component, which can have a different scale length. Here $M$ is the total mass of the galaxy. The interaction rate is given by the relative speeds of the solar system with respect to passing stars. The stars are expected to have random velocities superimposed on their overall orbital motion, where these random speeds are a fraction of the circular speed. Since both stars are moving, however, the relative speed is larger than the 
random speed. For purposes of this paper, we assume these effects cancel and use equation (3.7) to determine the relative speeds. In the future, a more detailed treatment of the relative velocities should be developed, along with considerations of the orbits through the galaxy [46].

\subsection{Survival Fractions for Scattering}

Equations (3.6) and (3.7) define the constraint that must be met in order for a solar system to survive over the time $t_{\mathrm{c}}$ (taken here to be $1 \mathrm{Gyr}$ ). To evaluate this constraint for a given galaxy mass $M$, we need to determine the stellar density scale $n_{0}$, and length scales $r_{\mathrm{dm}}$ and $r_{\mathrm{b}}$ for the dark matter halo and the baryonic component, respectively. Equation (2.18) specifies $n_{0}$, where we take the star formation efficiency $\epsilon_{\mathrm{sf}}=0.5$, the mean stellar mass $\left\langle M_{*}\right\rangle=0.5 M_{\odot}$, and the ratio $R_{\rho}=10$. This latter choice reflects the fact that baryons dissipate energy and can reach higher densities than the dark matter (and is roughly consistent with the relative densities in our galaxy [40]). We can then evaluate $n_{0}$ by using equation (2.5) to specify $\rho_{\mathrm{c}}$, which depends on the fluctuation amplitude $Q$. The scale length $r_{\mathrm{dm}}$ for the dark matter halo is then given by equation (2.13) and the corresponding scale for the baryonic component is given by equation (2.15). The remaining parameter that must be specified is the additional factor $f_{\text {vir }}$ that enhances the density (decreases the scale length). As reviewed in [8], $f_{\text {vir }} \sim 1$ for large halo masses near $M=M_{\text {eq }}$ and decreases to $f_{\text {vir }} \sim 0.03$ for a galactic mass halo $M \sim 2 \times 10^{12} M_{\odot}$ (where this latter value is close to the mass of the Local Group [47]). Here we model this additional collapse factor with a simple scaling law of the form $f_{\mathrm{vir}}=\left(M / M_{\mathrm{eq}}\right)^{3 / 5}$. Note that more sophisticated models can be used, where the density scale is written in terms of the halo concentration, which is then fit to numerical simulations [48].

In a galaxy with a given total mass $M$, a fraction of the constituent solar systems will reside at sufficiently large galactocentric radii to survive disruption of their planet orbits. Figure 2 shows the resulting survival fractions for galaxies with masses in the range $M=$ $10^{10}-10^{14} M_{\odot}$ (from lower left to top right in the figure). The survival fraction is shown as a function of the fluctuation amplitude $Q$. As the value of $Q$ increases, large scale structure forms earlier and the resulting structures are denser (characteristic of the background density of the universe at their formation epoch). Although solar systems are more easily disrupted for galaxies in universes with large $Q$, some fraction of the systems always survive. Note that we present results for density fluctuation amplitudes up to $Q \rightarrow 1$, but the formalism breaks down before reaching this limiting value (see the discussion of Section 6.2; see also [8]). For completeness, we also note that the number of surviving solar systems in a galaxy of mass $M$ is given by

$$
N_{\text {survive }}=F_{\text {survive }} \epsilon_{\mathrm{sf}} M /\left\langle M_{*}\right\rangle,
$$

where $\epsilon_{\mathrm{sf}}$ is the star formation efficiency and $\left\langle M_{*}\right\rangle$ is the mean stellar mass.

The survival fraction depends on galactic mass. Within the framework used here, galactic structures with smaller masses (e.g., $M=10^{10} M_{\odot}$, lower left curve) collapse to a greater degree, have smaller values of $f_{\text {vir }}$, and become more dense (see equation [2.5]). Even in the most unfavorable case, with the smallest galactic mass and the limiting value $Q \rightarrow 1$, the fraction of surviving solar systems is $\sim 0.003$, so that the galaxy is expected to have $\sim 3 \times 10^{6}$ viable systems. For a galaxy with a mass comparable to that of the Milky Way, $M=10^{12} M_{\odot}$ (center curve), the fluctuation amplitude must be larger than about $Q \gtrsim 0.01$ in order for more than half of the solar systems to be disrupted; in the limit $Q \rightarrow 1$, about 3 percent of the systems survive, corresponding to $\sim 3 \times 10^{9}$ viable solar systems per galaxy. 


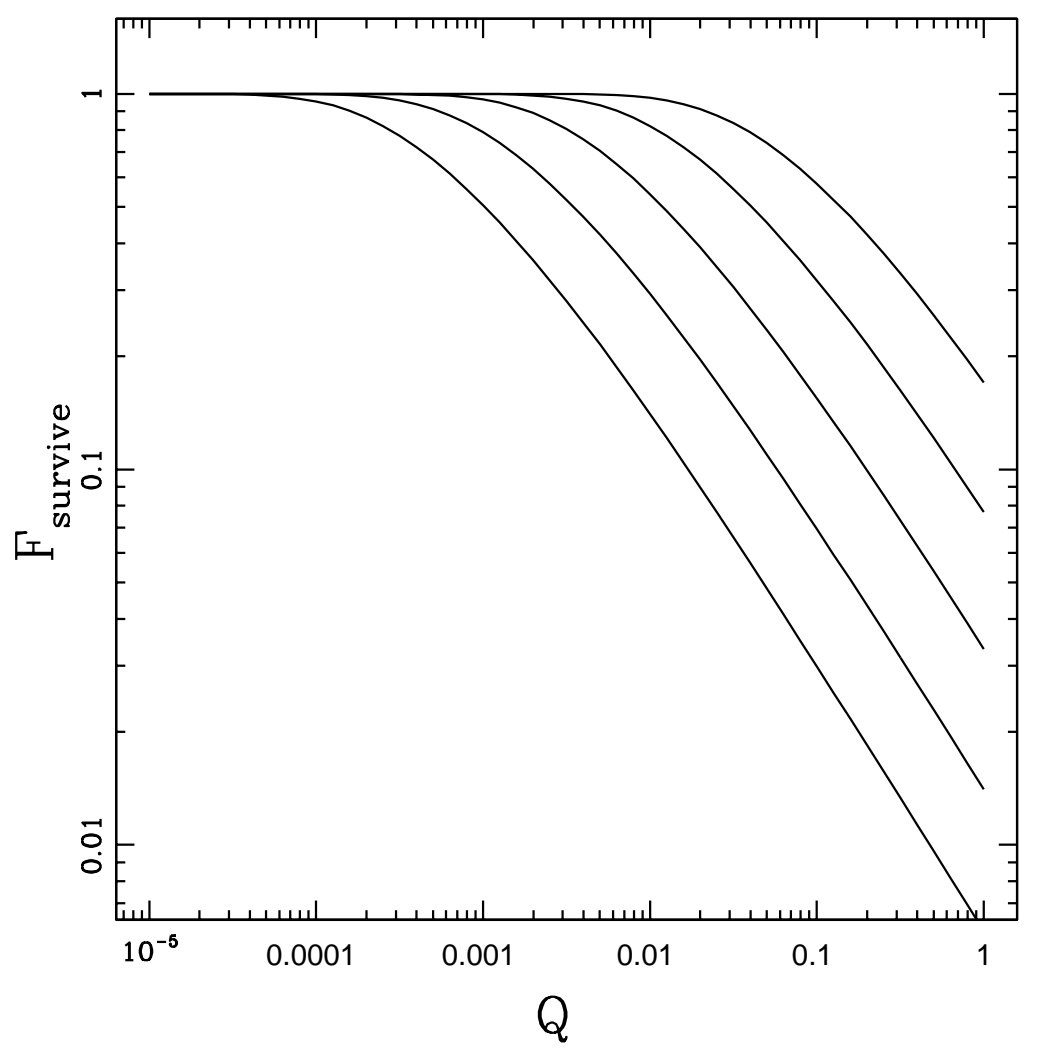

Figure 2. Fraction of solar systems that survive disruption from scattering encounters in galaxies of varying masses as a function of the fluctuation amplitude $Q$. In universes with larger $Q$, structure forms earlier, galactic structures are denser, and solar systems are more easily disrupted. The five curves shown correspond to galaxy masses $M=10^{10}-10^{14} M_{\odot}$ (from lower left to top right).

\section{Radiation Constraints}

In this section we find the radiation fields produced by the dense galaxies under consideration and estimate the fraction of solar systems that are disrupted through overheating. For this assessment, we assume that the interstellar medium of the galaxy is optically thin to its stellar radiation. This condition is expected to hold because these dense galaxies are likely to experience efficient star formation. If enough gas and dust remain in the galaxy, however, the interstellar medium could be optically thick, and the material would become even hotter in order to transport out the luminosity of the galaxy. This treatment thus represents a lower limit to the disruptive influence of the background radiation. On the other hand, we are implicitly assuming that the galaxy remains young enough that the stars have not yet burned out $[36,37]$.

To start, we consider a rough estimate for the effects of radiation. The total flux $F_{G}$ due to the background galaxy, at a particular location, is given by the sum over all of the constitutent stars,

$$
F_{G}=\sum_{\mathrm{j}=1}^{N} \frac{L_{j}}{4 \pi r_{j}^{2}},
$$


where $r_{j}$ is the distance from the given location to the $j t h$ star and $L_{j}$ is its luminosity. ${ }^{\dagger}$ To obtain an order of magnitude estimate, we first assume that the stars are distributed spherically. The total number of stars $N$ is then given by

$$
N=n_{*} \frac{4 \pi}{3} R^{3}
$$

where $R$ is the size of the stellar system. We can replace the sum in equation (4.1) by an integral, i.e.,

$$
F_{G}=\int_{0}^{R} 4 \pi r^{2} d r n_{*} \frac{\langle L\rangle}{4 \pi r^{2}}=n_{*}\langle L\rangle R,
$$

where $\langle L\rangle$ is the appropriate average value of the stellar luminosity (see the discussion below). In order for the background flux of the Galaxy to exceed that of the host star, we need

$$
n_{*}\langle L\rangle R>\frac{L}{4 \pi \varpi^{2}} \quad \text { or } \quad 4 \pi \varpi^{2} n_{*} R \frac{\langle L\rangle}{L}>1,
$$

where $\varpi$ is the orbital radius of the planet (assumed here to be in a circular orbit). We can eliminate $R$ in favor of the number $N$ of stars in the system,

$$
4 \pi \varpi^{2} n_{*}^{2 / 3}\left(\frac{3}{4 \pi}\right)^{1 / 3} N^{1 / 3} \frac{\langle L\rangle}{L}>1 .
$$

In the Galaxy today, this quantity is about $3 \times 10^{-6}$ (for the choice $\langle L\rangle / L=10$; see below). The radiation flux from the background galaxy thus becomes too intense for habitability when the stellar density is increased by a factor of $\sim 3 \times 10^{5}$ (note that this density enhancement is roughly comparable to that needed for scattering encounters to compromise planetary orbits, as found in Section 3).

\subsection{Radiation Fields within Galaxies}

In this section we construct the expected radiation fields for galaxies with extended structure, where we use the results from Section 2.2 to specify the density profiles. For a solar system located at the center of the galaxy, the radiation field from the background has the form

$$
F_{G}=\int_{0}^{\infty} 4 \pi r_{0}^{3} \xi^{2} d \xi \frac{n_{0}}{\xi(1+\xi)^{3}} \frac{\langle L\rangle}{4 \pi r_{0}^{2} \xi^{2}}=n_{0} r_{0}\langle L\rangle \int_{0}^{\infty} \frac{d \xi}{\xi(1+\xi)^{3}}
$$

where the scale length $r_{0}=r_{\mathrm{b}}$ is that of the baryonic component. Note that the integral is logarithmically divergent. As a result, for a solar system exactly at the galactic center, one must include a cutoff radius.

In practice, however, solar systems will not reside at the center. For a solar system with dimensionless radial position $\xi=a$, the expression for the background flux takes the form

$$
F_{G}=\frac{1}{2} n_{0} r_{0}\langle L\rangle \int_{0}^{\infty} \frac{\xi d \xi}{(1+\xi)^{3}} \int_{-1}^{1} \frac{d \mu}{\xi^{2}+a^{2}-2 \xi a \mu}
$$

\footnotetext{
${ }^{\dagger}$ Here and throughout the paper, $F_{G}$ is the magnitude of the total flux passing through the top of the atmosphere, and is evaluated at the location of the planet. Note that the flux of radiation outward through the galaxy is much smaller.
} 


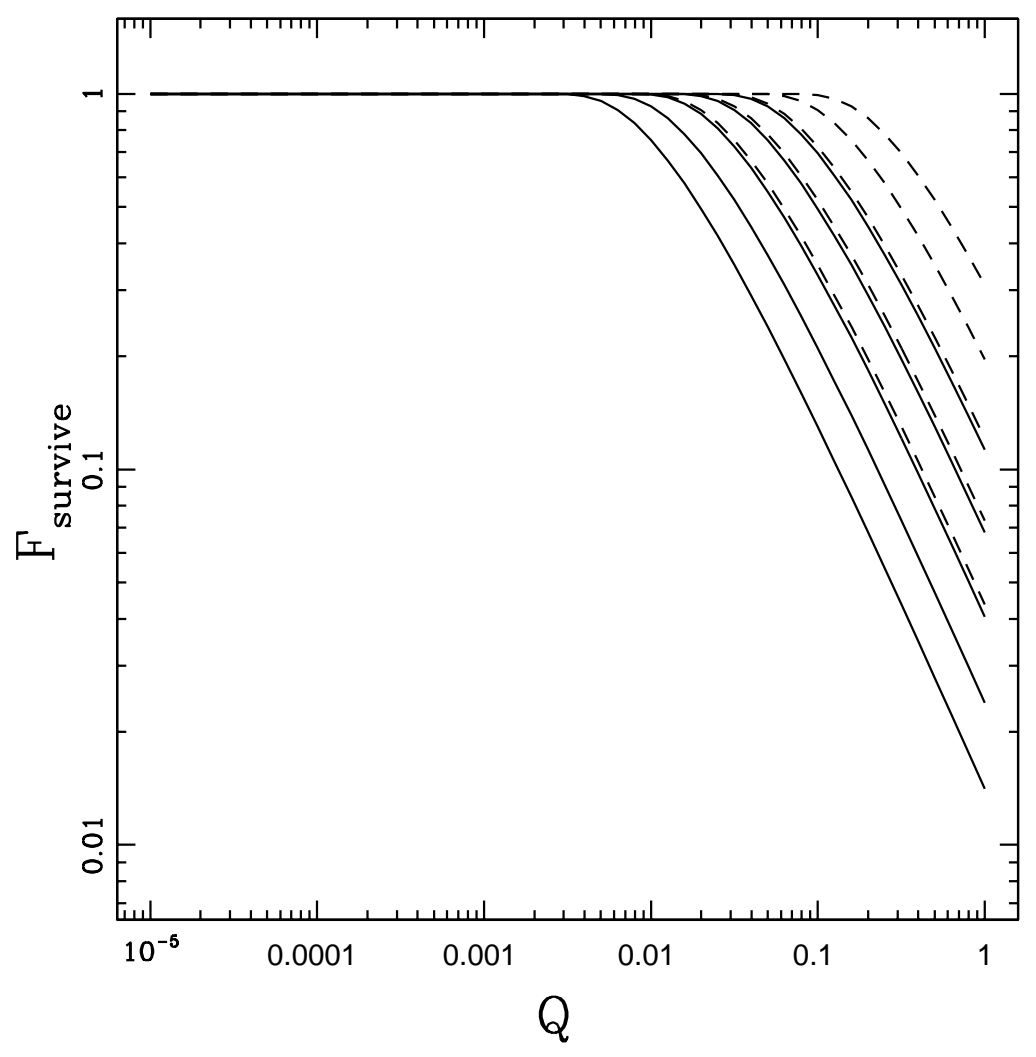

Figure 3. Fraction of solar systems that survive disruption from radiation in galaxies of varying masses as a function of the primordial fluctuation amplitude $Q$. As the value of $Q$ increases, structure forms earlier, so that galactic structures are denser and galactic background radiation is more intense. Results are shown for $\langle L\rangle / L=10$ (solid curves) and 1 (dashed curves). For each case, the five curves correspond to (total) galaxy masses $M=10^{10}-10^{14} M_{\odot}$ (from lower left to top right).

where $\mu=\cos \theta$ (and $\theta$ is the polar angle in spherical coordinates). The angular integral can be evaluated to obtain

$$
F_{G}=\frac{1}{4 a} n_{0} r_{0}\langle L\rangle \int_{0}^{\infty} \frac{d \xi}{(1+\xi)^{3}}\left\{\log \left[\xi^{2}+a^{2}+2 \xi a\right]-\log \left[\xi^{2}+a^{2}-2 \xi a\right]\right\} .
$$

The integral can be evaluated so the flux has the form

$$
F_{G}=\frac{n_{0} r_{0}\langle L\rangle}{2\left(a^{2}-1\right)}\left[1-\frac{2 \log a}{a^{2}-1}\right] .
$$

\subsection{Survival Fractions for Radiation}

Next we use the results outlined above to estimate the fraction of solar systems that will reside in regions of the galaxies where the background radiation field does not compromise habitability. The requirement that the radiation from the host star, at the location of the planet, exceeds that of the background galaxy takes the form

$$
\left(a^{2}-1\right)\left[1-\frac{2 \log a}{a^{2}-1}\right]^{-1}>n_{0} r_{0} 2 \pi \varpi^{2} \frac{\langle L\rangle}{L},
$$


where $a$ is the dimensionless radial location within the galaxy.

To evaluate this constraint, we need to specify the quantities on the right hand side of equation (4.10). For this estimate, the number density scale $n_{0}$ and length scale $r_{0}$ were determined as described in Section 3, where we considered survival due to planetary scattering. We also need to specify the relative brightness of the stellar population through the ratio $\langle L\rangle / L$, which measures the power output of the average star. In our present-day universe, the mass to light ratios for typical galaxies are of order $1-10$ in solar units [35, 40], where this accounting includes all contributions to the mass (i.e., dark matter, gas, and stellar remnants in addition to main-sequence stars). We thus expect the ratio $\langle L\rangle / L$ to be of order unity, or perhaps somewhat larger. Here we present results for $\langle L\rangle / L=1$ and 10 . Note that the corresponding light to mass ratio for zero-age main-sequence stars would imply a much larger ratio $\langle L\rangle / L \sim 1000$ [49]; the galactic average value, appropriate in this context, is lower because the high mass stars die out quickly and stellar remnants are included. We also note that the era of nuclear burning stars lasts for trillions of years [37], but not forever, so that the ratio $\langle L\rangle / L$ will eventually become much smaller.

Figure 3 shows the resulting fraction of solar systems that survive the intense radiation fields of their host galaxies, shown here as a function of the amplitude $Q$ of the primordial density fluctuations. These results are qualitatively similar to those obtained previously for disruption due to scattering of planetary orbits (compare with Figure 2). However, scattering interactions are somewhat more disruptive, so that the survival fractions shown in Figure 3 (considering the radiation fields) are higher. The most extreme case shown corresponds to the smallest galaxy with mass $M=10^{10} M_{\odot}$, the larger value of the luminosity ratio $\langle L\rangle / L$, and the limiting value of the fluctuation amplitude $Q \rightarrow 1$; even in this case, however, $1 \%$ of the solar systems are projected to survive the disruptive effects of radiation.

\subsection{Allowed Regions of Galactic Parameter Space}

The previous sections outline the requirements necessary for a solar system to survive disruption due to scattering by background stars (Section 3.3) and intense radiation fields (Section 4.2). These results show the fraction of surviving solar systems as a function of the fluctuation amplitude $Q$ (see Figures 2 and 3). In this section, we delineate the regions within a galaxy that allow solar systems to survive these two channels of disruption.

Survival from scattering requires that the scattering optical depth $\tau_{S}$ is less than unity,

$$
\tau_{S}=\frac{n_{0} \sigma v t_{\mathrm{c}}}{\xi(1+\xi)^{3}}<1 .
$$

Survival from radiation requires that the radiation received from the background galaxy is less than that of the host star, i.e.,

$$
\mathcal{R}=\frac{F_{G}}{F_{*}}=\frac{n_{0} r_{0} 2 \pi \varpi^{2}}{\xi^{2}-1} \frac{\langle L\rangle}{L}\left[1-\frac{2 \log \xi}{\xi^{2}-1}\right]<1 .
$$

Because planetary orbits can have different semimajor axes, this condition must be considered as approximate. Moreover, as considered in Section 5, the background galaxy could provide all of the power for a habitable planet. The constraint of equation (4.12) thus applies to conventional habitability.

Both the scattering optical depth $\tau_{S}$ and the radiation ratio $\mathcal{R}$ are functions of radial position $\xi$ within the galaxy. These functions are shown in Figure 4 for varying choices of the density scale $n_{0}$. The lower curves in Figure 4 correspond to values comparable to those 


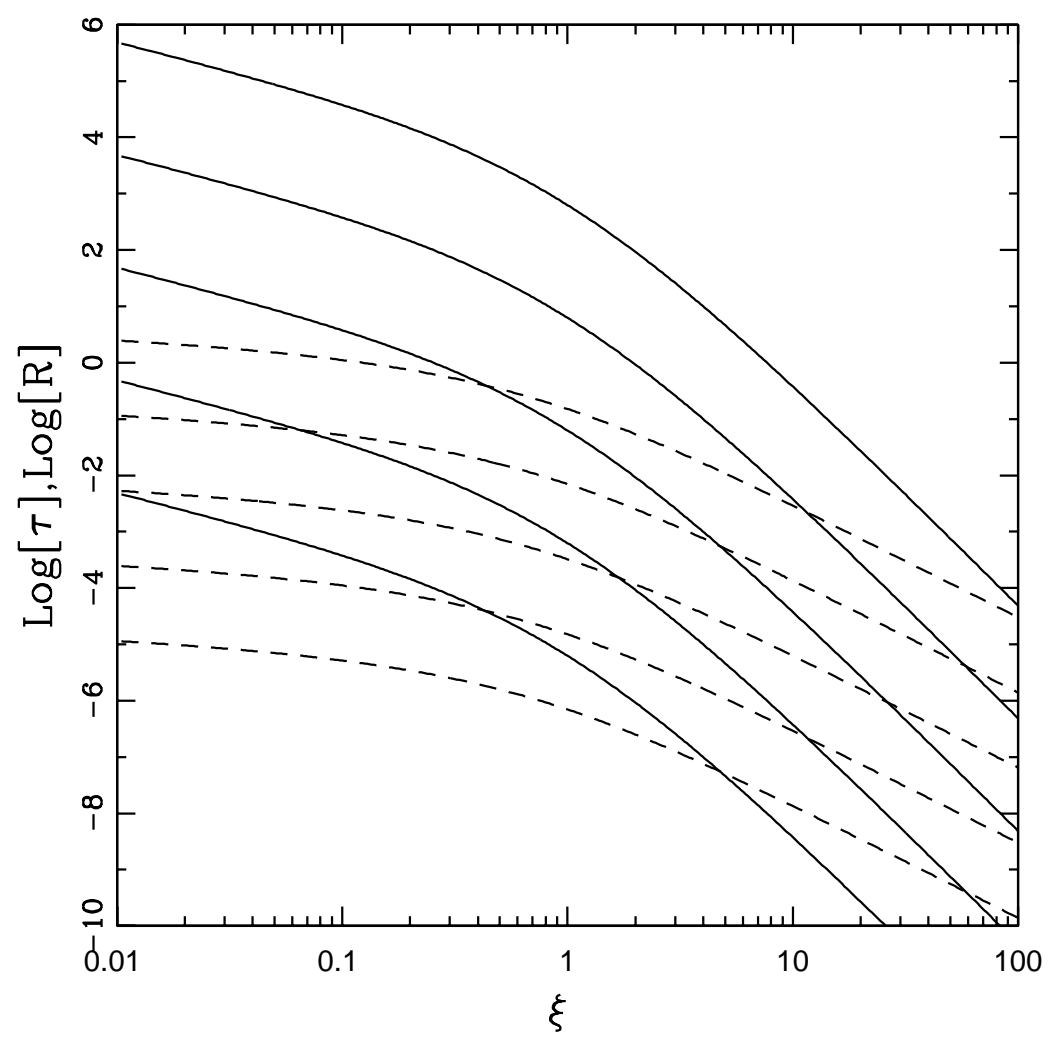

Figure 4. Disruption levels as a function of position within an idealized galaxy. The solid curves show the probablity of scattering an Earth-like planet (over $t_{\mathrm{c}}=1 \mathrm{Gyr}$ ) for galaxy models with varying density scales $n_{0}$. The dashed curves show the ratio of the radiation flux from the background galaxy to that provided by a central sun-like star. For both cases, curves are shown for galaxies with density scales $n_{0}=1$ (bottom), $10^{2}, 10^{4}, 10^{6}$, and $10^{8}$ (top), in units of $\mathrm{pc}^{-3}$.

of the solar neighborhood in the galaxy today. The remaining curves correspond to denser galactic environments, where the stellar density parameter is increased by factors up to $10^{8}$.

A number of trends are clear from Figure 4. First, for density enhancements of order $10^{6}$, the range of allowed parameter space starts to be compromised. Second, disruption by scattering interactions is usually more important than disruption by radiation. However, in the outer parts of the galaxy, the radiation fields provide the dominant channel of disruption. This behavior arises because the radiation flux decreases with radius as $1 / \xi^{2}$ whereas scattering disruption varies as $1 / \xi^{4}$ (where both results are evaluated in the limit $\xi \gg 1$ ). Finally, we note that the outer parts of the galaxy are always diffuse enough to allow survival in the face of both scattering and radiation, i.e., some fraction of the solar systems always survive.

Within the context of the formalism used here, the outskirts of the galaxy allow survival. In practice, however, the galaxy does not continue outward forever, but rather must have a most distant star. The fraction of stars contained within the dimensionless radius $\xi$ is given by

$$
\frac{N(\xi)}{N}=\frac{\xi^{2}}{(1+\xi)^{2}}=1-\frac{1+2 \xi}{(1+\xi)^{2}},
$$

where $N$ is the total number of stars. In order of magnitude, the outermost star in the galaxy 
resides at a radial location $\xi$ given by $N(\xi)=N-1$, or equivalently,

$$
\frac{1+2 \xi}{(1+\xi)^{2}}=\frac{1}{N}
$$

which has the solution

$$
\xi=N-1+\sqrt{(N-1)^{2}+N-1} \approx 2 N .
$$

In order for the galaxy to be so dense that even the outermost star experiences disruption through solar system scattering, the requirement becomes

$$
n_{0} \sigma v t_{\mathrm{c}}>(2 N)[1+2 N]^{3} \approx(2 N)^{4} .
$$

For a typical galaxy, the right hand side of this expression is $\sim 10^{44}$, so that the density scale $n_{0}$ must be larger than that of our galaxy by a factor of $\sim 10^{40}$ in order for no solar systems to survive. Using simple scaling arguments, the density $n_{0} \propto Q^{3}$, so that the largest enhancement factor is of order $10^{15}\left(\ll 10^{40}\right)$, so that galaxies are never dense enough to render all of their solar systems uninhabitable.

Note that the preceding calculation assumes that the stellar component of the galaxy does not have a well-defined outer edge. In practice, however, the outer parts of the galaxy can become too rarified to form stars [50], where the intergalactic radiation field prevents the formation of molecules. As a result, the outermost portions of the galaxy would have to be populated with stars via dynamical relaxation.

Before leaving this section, we note that scattering and radiation are roughly comparable as disruptive influences on habitable solar systems for galaxies in the present-day universe. We can understand this apparent coincidence as follows: The scattering optical depth is given by the product $n_{0} \sigma v t_{\mathrm{c}}$ (from equation [4.11]), whereas the analogous product that determines radiative disruption is $\sim n_{0} r_{0} \pi \varpi^{2}$ (from equation [4.12]). Both of these quantities thus depend linearly on the stellar density scale $n_{0}$. In addition, the cross section $\sigma$ for scattering is roughly comparable to the area of the planetary orbit $\pi \varpi^{2}$, which determines how much radiation is received from the central star. The coincidence is that the total distance traveled by a solar system during the benchmark time scale $\left(v t_{\mathrm{c}}\right)$ is comparable to the distance scale $r_{0}$ of the galactic density profile (i.e., the orbit time $r_{0} / v \sim t_{\mathrm{c}}$ ). More compact galaxies and especially their central regions - have shorter orbit times and are more disruptive via scattering (compared to radiation).

\section{$5 \quad$ Galactic Habitable Zones}

The intense radiation fields provided by compact galaxies introduce another possible channel for habitable planets. If the galactic background radiation has the proper intensity, then any potentially habitable planet can have the right temperature to support liquid water on its surface (one of the usual requirements for habitability [51]). More specifically, the surface temperature of a planet will have a minimum value, independent of its orbit within its host solar system (including unbound orbits). As expected, and as shown in Section 4, the background radiation flux within a galaxy decreases with galactocentric distance, so that a spherical shell within the galaxy will provide the proper radiations levels. We denote this region as the Galactic Habitable Zone (GHZ). 

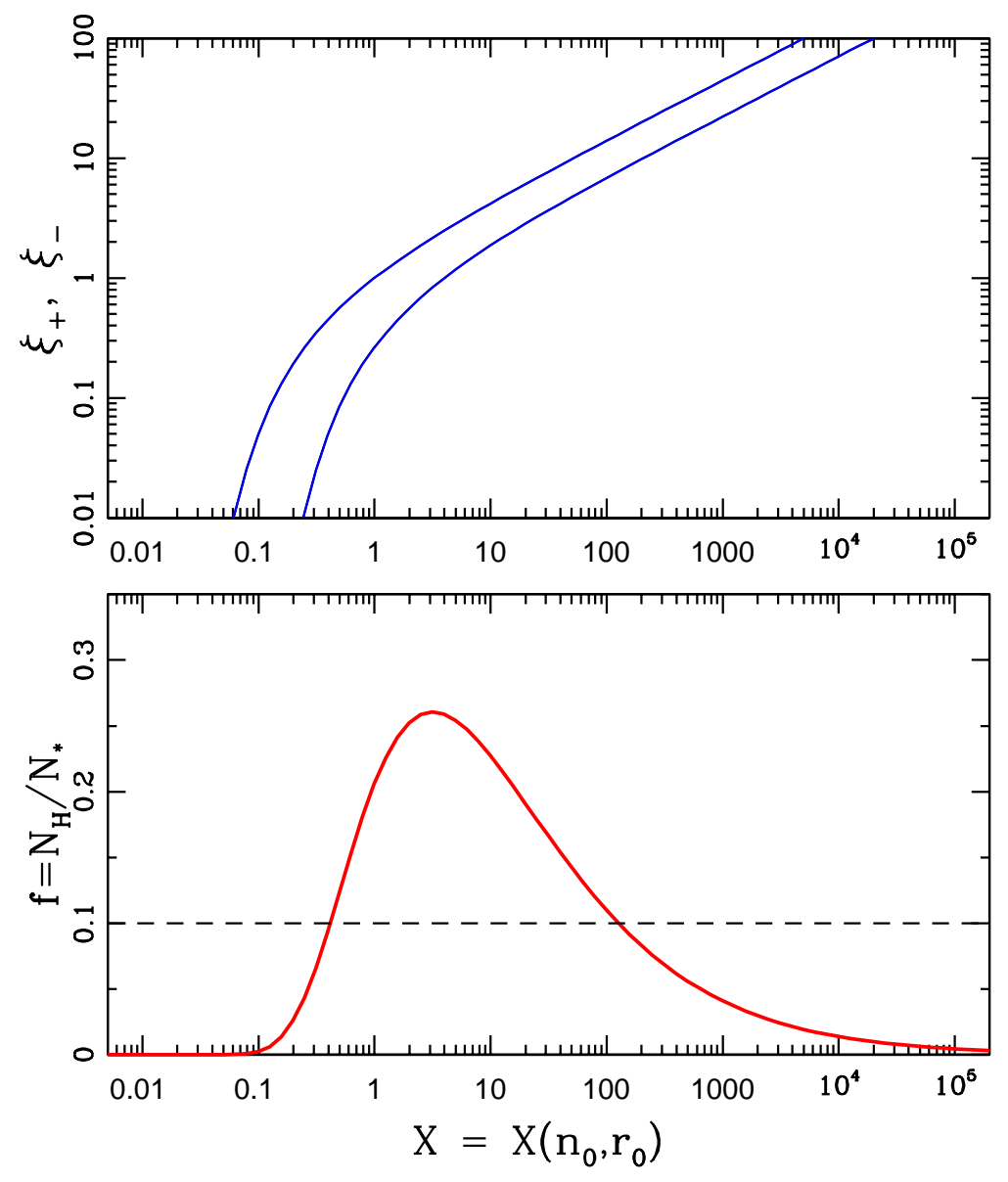

Figure 5. The Galactic Habitable Zone is the portion of a galaxy where the background radiation has the right intensity to support biospheres, independent of the orbital elements of the planet. The top panel shows the inner and outer boundaries of the GHZ as a function of the composite parameter $X$ that encapsulates the relevant galactic properites (see text). The bottom panel shows the fraction $f$ of the solar systems (per galaxy) that reside in the GHZ. If each star produces on average 10 planets, then galaxies with $f>0.1$ (above the dashed line) will support more potentially habitable planets than galaxies in our universe.

\subsection{Extent of the Galactic Habitable Zone}

We start by delineating the boundaries of the GHZ. Section 4 shows that the background galaxy provides a radiative flux $F_{G}$ given by equation (4.9). By equating this flux to that required for habitability, we obtain a constraint of the form

$$
F_{G}=C \frac{L_{\odot}}{4 \pi \varpi^{2}},
$$

where $\varpi$ is the semimajor axis of the Earth-analog orbit and the factor $C$ is a dimensionless constant of order unity. The requirement of liquid water on the surface of a planet depends on the planetary atmosphere and other properties. Here we use the requirement that the background flux of the galaxy $F_{G}$ is within a factor of two of that provided by the Sun to the Earth, i.e., we allow $C$ to vary over the range $0.5 \leq C \leq 2$. Next we define a composite 
parameter $X$ that incorporates the galactic properties,

$$
X \equiv 2 \pi \varpi^{2} n_{0} r_{0} \frac{\langle L\rangle}{L_{\odot}} \approx 1.57\left(\frac{n_{0}}{10^{6} \mathrm{pc}^{-3}}\right)\left(\frac{r_{0}}{1 \mathrm{kpc}}\right)\left(\frac{\langle L\rangle}{10 L \odot}\right) .
$$

The Galactic Habitable Zone then lies between radial locations $\xi=\xi_{ \pm}$that statify the requirement

$$
\frac{1}{\xi^{2}-1}\left[1-\frac{2 \log \xi}{\xi^{2}-1}\right]=\frac{C}{X},
$$

where $C=1 / 2$ for the outer boundary and $C=2$ for the inner boundary of the GHZ.

The resulting boundaries of the GHZ are shown in the upper panel of Figure 5 as a function of the composite parameter $X$. The corresponding fraction $f=N_{H} / N_{*}$ of solar systems that reside in the GHZ are shown in the lower panel of the figure. The horizontal dashed line delineates the portion of parameter space for which 10 percent of the solar systems reside in the GHZ, which corresponds to values of the parameter $X$ in the range $X \approx 0.4-125$. Note that nearly all of the planets in the GHZ are potentially habitable, by definition. In contrast, within ordinary galaxies (like our own), only a fraction of planets have orbits at the right distance from their host stars to support biospheres. Moreover, this fraction is only about 10 percent: The observed orbital spacing in solar systems is such that only about one star per solar system has the right temperature, and solar systems can often support $\sim 10$ planets. This result raises an interesting possibility: Galaxies with composite parameter $X=1-100$ could support more habitable planets than those in our universe.

The condition of having a robust GHZ requires the parameter $X$ to lie in the range 1 -100 , which requires that the column density of stars in the galaxy $n_{0} r_{0} \approx 10^{8}-10^{10} \mathrm{pc}^{-2}$ (where we take $\langle L\rangle / L=1$ ). For comparison, typical column densities for galaxies in our universe are of order $n_{0} r_{0} \sim 100$ (e.g., for a galaxy with $N=10^{11}$ stars and scale length $r_{0}$ $=12.5 \mathrm{kpc}$ ). As another benchmark, the surface brightness of our Galaxy is $\sim 1000 L_{\odot} \mathrm{pc}^{-2}$ [40], which converts to a column density in stars of order $100-1000 \mathrm{pc}^{-2}$. As a result, within the context of this formalism, the most favorable galaxies for potentially habitable planets must have column densities enhanced (relative to our universe) by factors of $10^{6}-10^{8}$.

We would like to find the range of fluctuation amplitudes $Q$ that produces galaxies with large GHZs. Unfortunately, there is not a definitive one-to-one mapping between the constraints on galactic properties and analogous constraints on the density fluctuation parameter $Q$ : Universes with a given $Q$ produce galaxies over a range of mass scales, and different galactic masses collapse to a range of densities and sizes. Nonetheless, we can make a rough estimate using typical values for the galactic masses. Since we expect the density to scale with fluctuation amplitude according to $n_{0} \propto Q^{3}$ and the length parameter to scale according to $r_{0} \propto 1 / Q$, the column density scales as $Q^{2}$. The preferred fluctuation amplitude $Q_{\text {opt }}$ for habitable planets is thus larger than that of our universe by a factor of $10^{3}-10^{4}$, so that the optimum amplitude is expected to fall in the range

$$
Q_{\mathrm{opt}}=0.01-0.1 \text {. }
$$

The above scaling argument indicates that a substantial GHZ requires relatively large values of the amplitude $Q$. In such universes, however, several complications should be kept in mind: Large fluctuation amplitudes can lead to rapid structure formation, and could increase the nonlinear mass scale beyond that realized in our Universe. If the component of dark energy - which acts to accelerate the universe - is sufficiently small, then galaxies can 
readily merge with each other, and the first generation of galaxies would not survive long enough to support life. The question of habitability would then depend on the nature of the resulting merged galactic structures. In addition, the central regions of these dense galaxies are likely to support the formation of massive black holes, which would alter the galactic luminosity.

Some authors have suggested that the probability of an inflationary universe producing a given amplitude for the density fluctuations is an increasing function of $Q$ [7]. This result is consistent with the finding that small values of $Q$ require carefully chosen small parameters in the inflationary potential $[5,6]$. As a result, universes with larger values of $Q$, including the range indicated in equation (5.4), could be more common than our own.

\subsection{Properties and Consequences}

For the larger stellar densities expected within the GHZ, planets have a good chance of being scattered out of their orbits. Over the range of galactic masses and the amplitudes $Q_{\mathrm{opt}}$ given in equation (5.4), anywhere from $3 \%$ to $97 \%$ of the planets in Earth-like orbits will experience disruption (see Figure 2). Planets on wider orbits will be disrupted to an even greater extent. As a result, the majority of the viable planet population in this scenario will be freely floating, rather than bound in orbit about their host stars. Although scattering encounters are expected to liberate planets in the GHZ, stellar collisions will be rare for time scales $t_{\mathrm{c}}$. At the high densities of the galactic centers, however, stellar collisions can more readily take place. The centers of these galaxies are thus favorable sites for the formation of large black holes [52].

Freely floating planets, and entire solar systems, can eventually leave the GHZ through accumulated perturbations from passing stars. The time required for this process of dynamical relaxation is given by

$$
t_{\text {relax }}=\frac{v^{3}}{8 \pi \Lambda G^{2} M_{*}^{2} n_{*}},
$$

where $v$ is the orbit speed and $\Lambda \equiv \ln \left[b_{2} / b_{1}\right]$, where $b_{1}$ and $b_{2}$ are the minimum and maximum possible impact parameters [35]. Using equation (5.5) to evaluate the relaxation time for galaxies with $X=1-100$ and for locations within the GHZ, we find

$$
t_{\text {relax }} \sim 10^{5} \operatorname{Gyr}\left(\frac{N_{*}}{10^{12}}\right)^{1 / 2}\left(\frac{r_{0}}{0.1 \mathrm{kpc}}\right)^{3 / 2} \xi^{5 / 2},
$$

where $N_{*}$ is the total number of stars in the galaxy. Since the dimensionless radius $\xi \sim 1$ in the GHZ, the relaxation time is much longer than the characteristic time scale $t_{\mathrm{c}}=1 \mathrm{Gyr}$ required for habitability. As a result, most potentially habitable planets will stay within the GHZ long enough for biological evolution to take place. ${ }^{\dagger}$

In a related but different context, the concept of a Galactic Habitable Zone has been put forth for our Galaxy [53]. In that case, however, the conditions required to compromise habitability are much less extreme than those of this paper. Specifically, the habitable zone from [53] is based on the chemical makeup of the galaxy as a function of position (and requires the metallicity to be within a factor of $\sim 2$ of the value for our Solar System). Since the metallicity $Z$ decreases with galactocentric distance, the outer parts of the galaxy have low $Z$ and are less suited for life. The definition of the GHZ used in this paper is similar in

\footnotetext{
${ }^{\dagger}$ Note that the central regions of the galaxies, where $\xi \ll 1$, have much shorter relaxation times, so that rearrangement of stellar orbits is expected there.
} 
spirit to the constraints of [54], who considered the disruption of planetary systems in rich star clusters. In this latter study, sufficiently rich stellar environments produce intense radiation fields that heat planet-forming disks above the temperature required for ices to condense; with no ices, the process of giant planet formation can be compromised.

This present work focuses on the survival of planetary systems after they are made; future studies should also consider how environmental effects can hinder their formation. In particular, the background temperature within the GHZ allows for water to be in liquid form at atmospheric pressures, which implies that the water could be gaseous at the lower pressures characteristic of planet-forming disks [55]. In this case, the dust grains - and later the rocks - that provide the building blocks for planets will have little water, and terrestrial planets could end up dry. Note that this dilemma is a scaled up version of the same issue facing potentially habitable planets in our universe: In the inner solar system, where planets are warm enough to support biospheres, the rocky planets must be built out of raw material with relatively little water. As a result, alternate channels for the delivery of water to Earth, and hence other terrestrial planets, have been considered. If enough of the nebula remains intact after a terrestrial planet is formed, its atmosphere can be both massive and rich in hydrogen. In this setting, the atmospheric hydrogen can react with various oxides (such as $\mathrm{FeO}$ ) in the still-liquid rocky planet and produce a significant amount of water $[56,57]$. In any case, the delivery of water to potentially habitable planets in the GHZ should be explored further.

For completeness, we note that the radiation fields found in the GHZ are much less intense than those required to make the night sky as bright as a stellar surface, the condition considered in Olbers' paradox [58, 59]. In order for any given line of sight to intercept a stellar surface, the optical depth through the galaxy would have to exceed unity, so that $n_{0} r_{0} \pi R_{*}^{2} \sim 1$ (compare with the analogous expressions for disruption from equations [4.11] and [4.12]). However, stellar radii $R_{*}$ are much smaller than planetary orbits ( $\left.\varpi \sim 1 \mathrm{AU}\right)$ or scattering cross section length scales $(\ell \sim \sqrt{\sigma} \sim 1 \mathrm{AU})$. As a result, the column density $n_{0} r_{0}$ of stars through the galaxy would have to be much larger than the values considered here, by a factor of $\left(\varpi / R_{*}\right)^{2} \sim 4 \times 10^{4}$, for the night sky to be as bright as the surface of the Sun. ${ }^{\dagger}$ This distinction is important: The incoming radiation has the energy characteristic of stellar photospheres (with temperatures $T_{*} \approx 3000-10,000 \mathrm{~K}$ ), whereas the planets have much lower temperatures $T_{P} \approx 273-373 \mathrm{~K}$ (and emit lower energy, infrared radiation). This temperature difference allows for a heat engine to operate and thereby perform the work necessary to run a biosphere.

In order for a biosphere to operate, the heat generated by the planet must be able to radiate away. In our present-day universe, the background temperature of the galaxy $\left(T_{\text {gal }} \sim 3-10 \mathrm{~K}\right)$ is much colder than the planetary temperature $T_{P}$, so that exhaust heat poses no problem. Since the background temperature of the galaxy is hotter in the GHZ, one might worry that a heat engine could have trouble operating. As outlined above, however, the galaxy is optically thin to its internal radiation, including both stellar radiation and that re-radiated by planets. As a result, the ultimate heat sink for the galaxy is cold extragalactic space (where $\left.T \ll T_{P}\right)$. Planets can thus absorb optical light and freely re-radiate the energy as infrared radiation. This process generates large amounts of entropy, which allows for a decrease in the entropy in parts of the biosphere itself. In addition, planetary atmospheres raise the temperature of the planets further, and this increase also helps to radiate the necessary exhaust heat. For completeness, we also note that a biosphere does not function as a simple

\footnotetext{
${ }^{\dagger}$ In other words, in the GHZ, the night sky is (approximately) as bright as the daytime sky on Earth, but not as bright as a stellar photosphere.
} 
carnot heat engine, so that its efficiency is not given by the classical expression $\left(\epsilon=1-T_{c} / T_{h}\right)$. Instead, one must consider the Gibbs free energy of the system, including all of the terms associated with the different particle components and their chemical potentials (for further discussion, see $[42,43])$.

\section{Conclusion}

Extending previous work, this paper has shown that when galactic stellar systems become sufficiently dense relative to those in our present-day universe, significant disruption of solar systems can take place. Such disruption can render Earth-like planets uninhabitable through two different mechanisms: Scattering interactions can perturb planetary orbits before life has time to evolve and radiation from the background galaxy can dominate that of the host star and thereby make all planets too hot to support biospheres. Nonetheless, some fraction of the potentially habitable systems will survive. A summary of our results is given below (in Section 6.1), followed by a discussion of their implications and indications for further work (Section 6.2).

\subsection{Summary of Results}

We have performed a large ensemble of $\sim 10^{6}$ numerical simulations to determine the interaction cross sections for the disruption of planetary orbits via encounters with passing stars (see Figure 1). The disruption cross section of Earth-analog orbits can be fit with the function given by equation (3.3). As expected, this cross section approaches the asymptotic form $\sigma \sim 1 / v^{2}$ in the high speed limit, where the limiting regime requires $v \gg 1000 \mathrm{~km} \mathrm{~s}^{-1}$.

The density of stars within a galaxy is a function of position; here we use the Hernquist profile of equation (2.11) as a working model. For this class of profiles, both the scattering rate $\Gamma$ and the background radiation flux $F_{G}$ diverge in the inner limit $\xi \rightarrow 0$. On the other hand, both $\Gamma \rightarrow 0$ and $F_{G} \rightarrow 0$ in the limit of large radii $\xi \gg 1$. Inclusion of galactic structure thus changes the problem from previous treatments, which implicitly assume that galaxies are characterized by a single density. In every galaxy, some fraction of the solar systems will be disrupted by scattering encounters, whereas some other fraction will be disrupted by background radiation fields (and some fraction will survive).

The characteristic densities for galactic structure (see equation [2.5]) increase with the amplitude $Q$ of the primordial density fluctuations. As a result, the fraction of solar systems within a galaxy that survive disruption - and thereby remain potentially habitable - is a decreasing function of $Q$. This trend is shown in Figure 2 for disruption of planetary orbits by scattering encounters and in Figure 3 for disruption by the background radiation field of the galaxy. Note that the survival fractions also depend on galactic mass (as shown in the figures). The outer parts of the galaxies remain habitable for essentially any choice of the galactic structure parameters (see Figure 4).

Because of the intense galactic background radiation fields, some portion of the galaxy can provide the right flux levels to support habitable planets. Any planets residing in this region - denoted here as the Galactic Habitable Zone - can have surface temperatures compatible with maintaining liquid water. Although planets residing too close to their host stars will still be too hot, planets in all other orbits, including freely floating bodies, can in principle support biospheres. Moreover, the GHZ is large enough so that some galaxies in other universes can support more potentially habitable planets (powered by the background radiation of the galaxy) than the galaxies in our present-day universe (Figure 5). 


\subsection{Discussion}

In approximate terms, disruption of habitable planets through both scattering and radiation becomes important when the background density of stars is a million times larger than that of the Solar Neighborhood, which is not atypical within galaxies of our present-day universe. Since galactic densities increase with the approximate scaling $\rho \propto Q^{3}$ (equation [2.5]), we naively expect the disruption of habitable planets to become important when $Q$ is about 100 times larger than in our universe, i.e., for $Q \gtrsim 10^{-3}$. This expectation is borne out in Figures 2 and 3, although universes can remain habitable for even larger values of $Q$ if we only require that a fraction of the solar systems remain viable.

The results of this work indicate that habitable planets can survive in galaxies with a wide range of properties, and hence in a wide range of universes. At first glance, this result may seem to contradict previous work that places relatively tight limits on the fluctuation amplitude $Q$. In this current generalized treatment, however, more planets can survive for several reasons: [A] Galactic structures naturally include a wide range of stellar densities, with lower densities in the outer regimes that allow for habitable orbits to survive. [B] With the consideration of internal galactic structure, we can determine the fraction of solar systems that avoid disruption (rather than using an all-or-nothing approach). Because planets are thought to be common, and galaxies contain many solar systems, a galaxy (universe) can remain habitable with only a small fraction of its solar systems surviving (note that Figures 2 and 3 are plotted with logarithmic scales). [C] Even planets that are stripped from their host stars can remain habitable if they reside within the Galactic Habitable Zone, i.e., the region of the galaxy where the galactic background radiation has the proper intensity to heat planets.

The results of this paper are presented for density fluctuation amplitudes as large as $Q=1$. However, the reader should keep in mind that the formalism breaks down before reaching such high values. For sufficiently large fluctuation amplitudes, structure formation is thought to take place violently, thereby leading to rampant black hole formation and other departures from the standard scenario. This regime of structure formation has not been well-studied via numerical simulations, but such work should be carried out in the future. Even for the regime of moderately higher values of the amplitude $Q$, numerical simulations of structure formation should be carried out in order to verify the assumption that dark matter halos approach a nearly universal form (assumed here to be NFW/Hernquist). We also need a better specification of what happens to the baryons in these high- $Q$ scenarios. In this work, we assume that galaxies become scaled-up versions of the galactic bulges in our universe (with density profiles of equation [2.11]). If the galactic system has enough angular momentum, however, baryons can settle into a rotationally supported disk structure (like the spiral galaxies of our universe). The survival fractions and background radiation fields should also be determined for galaxies with disk geometries.

This treatment considers universes with different amplitudes $Q$ for the density fluctuations, and hence denser galactic structures, but holds fixed the other properties of the universe. In general, however, both the fundamental constants of physics and cosmological variables (in addition to $Q$ ) could be different in other universes. Such additional variations would change the predicted fraction of habitable solar systems. As one example, if the gravitational constant $G$ is larger, then stars are brighter [60-62], and habitable planets must have larger orbits; as a result, planetary scattering could be enhanced. On the other hand, this change could be offset with an increase in the fine structure constant $\alpha$, which would act to suppress nuclear fusion and would require closer orbits to achieve chemical reactions [20,63]. Future 
work should thus take into account coupled variations in all of the relevant parameters. In addition, the 'constants' of nature could also vary with time within a particular universe $[64,65]$. Considering all of the possibilities is an enormous undertaking; the first step is to consider more limited possible variations as carried out here.

Finally, we note that most considerations of alternate universes use the properties of our own universe as the baseline for comparison, often with an implicit assumption that our universe provides the best possible environment for the development of life. The results of this paper call this assumption into question: With the right parameters, galaxies can support extensive Galactic Habitable Zones, where up to $20 \%$ of the stars, and hence up to $20 \%$ of all planets, reside within a radiation field comparable to the one the Earth receives from our Sun. Such galaxies - residing in universes with the right properties - could support more habitable planets than our own. Within our universe, Earth is often considered as the optimal planet for supporting a biosphere, but other planets could be even more favorable [66]. This issue, which could be called superhabitability, should also be explored further.

\section{Acknowledgments}

We would like to thank Konstantin Batygin, Juliette Becker, Gus Evrard, Evan Grohs, Gordy Kane, Minhyun Kay, Jake Ketchum, and Chris Spalding for useful discussions and suggestions, as well as two anonymous referees. This work was supported by JT Foundation grant ID55112 "Astrophysical structures in other universes" and by the University of Michigan. FCA is also supported by FQXi grant MGB-1414; AMB is also supported by NSF grants INSPIRE1363720 and DMS-1207693, and by the Simons Foundation.

\section{References}

[1] G. F. Smoot et al., Structure in the COBE Differential Microwave Radiometer First-year Maps, Astrophys. J. 396 (1992) L1

[2] D. N. Spergel et al., Three-Year Wilkinson Microwave Anisotropy Probe (WMAP) Observations: Implications for Cosmology, Astrophys. J. Suppl. 170 (2007) 377

[3] Planck Collaboration: P.A.R. Abe et al., Planck 2013 Results. XVI: Cosmological parameters, Astron. Astrophys. 571 ( 2014) 16

[4] A. H. Guth, Inflation and Eternal Inflation, Phys. Rep. 333 (2000) 555

[5] J. M. Bardeen, P. J. Steinhardt, and M. S. Turner, Spontaneous Creation of Almost Scale-free Density Perturbations in an Inflationary Universe, Phys. Rev. D 28 (1983) 679

[6] F. C. Adams, K. Freese, and A. H. Guth, Constraints on the Scalar-field Potential in Inflationary Models, Phys. Rev. D 43 (1991) 965

[7] J. Garriga and A. Vilenkin, Anthropic Prediction for $\Lambda$ and the $Q$ Catastrophe, Prog. Theor. Phys. 163 (2006) 245

[8] M. Tegmark and M. J. Rees, Why Is the Cosmic Microwave Background Fluctuation Level $10^{-5}$ ? Astrophys. J. 499 (1998) 526

[9] M. Tegmark, A. Aguirre, M. J. Rees, and F. Wilczek, Dimensionless Constants, Cosmology, and other Dark Matters, Phys. Rev. D 73 (2006) 3505

[10] M. J. Rees and J. P. Ostriker, Cooling, Dynamics and Fragmentation of Massive Gas Clouds: Clues to the masses and radii of galaxies and clusters, Mon. Not. R. Astron. Soc. 179 (1977) 541 
[11] S.D.M. White and M. J. Rees, Core Condensation in Heavy Halos: A two-stage theory for galaxy formation and clustering, Mon. Not. R. Astron. Soc. 183 (1978) 341

[12] M. J. Rees, Before the Beginning, Perseus (1997)

[13] M. J. Rees, Just Six Numbers, Basic Books (2000)

[14] A. Vilenkin, Unambiguous Probabilities in an Eternally Inflating Universe, Phys. Rev. Lett. 81 (1998) 5501

[15] B. J. Carr and M. J. Rees, The Anthropic Principle and the Structure of the Physical World, Nature 278 (1979) 611

[16] C. J. Hogan, Why the Universe is Just So, Rev. Mod. Phys. 72 (2000) 1149

[17] A. Aguirre and M. Tegmark, Multiple Universes, Cosmic Coincidences, and other Dark Matters, J. Cosmol. Astropart. Phys. 01 (2005) 003

[18] M. Tegmark, What does Inflation Really Predict? J. Cosmol. Astropart. Phys. 04 (2005) 001

[19] L. A. Barnes, The Fine-Tuning of the Universe for Intelligent Life, Pub. Astron. Soc. Australia 29 (2012) 529

[20] J. D. Barrow and F. J. Tipler, The Anthropic Cosmological Principle, Oxford Univ. Press (1986)

[21] V. F. Weisskopf, Of Atoms, Mountains, and Stars: A study in qualitative physics, Science 187 (1975) 605

[22] E. V. Quintana, T. Barclay, S. N. Raymond, et al., An Earth-Sized Planet in the Habitable Zone of a Cool Star, Science 344 (2014) 277

[23] E. A. Petigura, A. W. Howard, and G. W. Marcy, Prevalence of Earth-size Planets orbiting Sun-like Stars, Proc. Nat. Acad. Sci. 110 (2013) 19273

[24] J. E. Gunn and J. R. Gott III, On the Infall of Matter Into Clusters of Galaxies and Some Effects on Their Evolution, Astrophys. J. 176 (1972) 1

[25] W. H. Press and P. Schecter, Formation of Galaxies and Clusters of Galaxies by Self-Similar Gravitational Condensation, Astrophys. J. 187 (1974) 425

[26] E. W. Kolb and M. S. Turner, The Early Universe, Addison-Wesley (1990)

[27] K. Nagamine and A. Loeb, Future Evolution of Nearby Large-scale Structures in a Universe Dominated by a Cosmological Constant, New Astron. 8 (2003) 439

[28] M. T. Busha, A. E. Evrard, F. C. Adams, and R. H. Weschler, The Ultimate Halo Mass in a $\Lambda C D M$ Universe, Mon. Not. R. Astron. Soc. 363 (2005) L11

[29] M. T. Busha, A. E. Evrard, and F. C. Adams, The Asymptotic Form of Cosmic Structure: Small-Scale Power and Accretion History, Astrophys. J. 665 (2007) 1

[30] J. F. Navarro, C. M. Frenk, and S.D.M. White, A Universal Density Profile from Hierarchical Clustering, Astrophys. J. 490 (1997) 493

[31] L. Hernquist, An Analytical Model for Spherical Galaxies and Bulges, Astrophys. J. 356 (1990) 359

[32] M. Surhud, B. Diemer, and A. V. Kravtsov, arXiv:1504.05591 (2015)

[33] S. Cannizzo and T. C. Hollister, Cold Dissipationless Collapse of Spherical Systems: Sensitivity to the initial density law, Astrophys. J. 400 (1992) 58

[34] C. M. Boily and E. Athanassoula, On the Equilibrium Morphology of Systems Drawn from Spherical Collapse Experiments, Mon. Not. R. Astron. Soc. 369 (2006) 608

[35] J. Binney and S. Tremaine, Galactic Dynamics, Princeton Univ. Press (2008) 
[36] F. J. Dyson, Time Without End: Physics and biology in an open universe, Rev. Mod. Phys. 51 (1979) 447

[37] F. C. Adams and G. Laughlin, A Dying Universe: The long-term fate and evolution of astrophysical objects, Rev. Mod. Phys. 69 (1997) 337

[38] J. Binney, Resonant Excitation of Motion Perpendicular to Galactic Planes, Mon. Not. R. Astron. Soc. 196 (1981) 455

[39] F. C. Adams, A. M. Bloch, S. C. Butler, J. M. Druce, and J. A. Ketchum, Orbital Instabilities in a Triaxial Cusp Potential, Astrophys. J. 670 (2007) 1027

[40] J. Binney and M. Merrifield, Galactic Astronomy, Princeton Univ. Press (1998)

[41] G. Laughlin and F. C. Adams, The Frozen Earth: Binary Scattering Events and the Fate of the Solar System, Icarus 145 (2000) 614

[42] J. I. Lunine, Astrobiology: A Multidisciplinary Approach, Pearson (2005)

[43] C. A. Scharf, Extrasolar Planets and Astrobiology, Univ. Science Books (2009)

[44] F. C. Adams and G. Laughlin, Constraints on the Birth Aggregate of the Solar System, Icarus 150 (2001) 151

[45] G. Li and F. C. Adams, Cross-sections for Planetary Systems Interacting with Passing Stars and Binaries, Mon. Not. R. Astron. Soc. 448 (2015) 344

[46] F. C. Adams and A. M. Bloch, Orbits in Extended Mass Distributions: General Results and the Spirographic Approximation, Astrophys. J. 629 (2005) 204

[47] J. Peñarrubia, Y.-Z. Ma, M. G. Walker, and A. McConnachie, A Dynamical Model of the Local Cosmic Expansion, Mon. Not. R. Astron. Soc. 443 (2014) 2204

[48] F. Prada, A. A. Klypin, A. J. Cuesta, J. E. Betancort-Rijo, and J. Primack, Halo Concentrations in the Standard $\Lambda$ Cold Dark Matter Cosmology, Mon. Not. R. Astron. Soc. 423 (2012) 3018

[49] C. Leitherer et al., Starburst99: Synthesis Models for Galaxies with Active Star Formation, Astrophys. J. Suppl. 123 (1999) 3

[50] J. Schaye, Star Formation Thresholds and Galaxy Edges: Why and Where, Astrophys. J. 609 (2004) 667

[51] J. F. Kasting, D. P. Whitmire, and R. T. Reynolds, Habitable Zones around Main Sequence Stars, Icarus 101 (1993) 108

[52] M. J. Rees, Black Hole Models for Active Galactic Nuclei, Ann. Rev. Astron. Astrophys. 22 (1983) 471

[53] G. Gonzalez, D. Brownlee, and P. Ward, The Galactic Habitable Zone: Galactic Chemical Evolution, Icarus 152 (2001) 185

[54] T. A. Thompson, Gas Giants in Hot Water: Inhibiting giant planet formation and planet habitability in dense star clusters through cosmic time, Mon. Not. R. Astron. Soc. 431 (2013) 63

[55] C. Hayashi, Structure of the Solar Nebula, Growth and Decay of Magnetic Fields and Effects of Magnetic and Turbulent Viscosities on the Nebula, Prog. Theor. Phys. Suppl. 70 (1981) 35

[56] S. Sasaki, in Origin of the Earth, eds. H. E. Newsom and J. H. Jones, Oxford Univ. Press) (1990) p. 195

[57] M. Ikoma and H. Genda, Constraints on the Mass of a Habitable Planet with Water of Nebular Origin, Astrophys. J. 648 (2006) 696

[58] E. R. Harrison, Olbers' Paradox and the Background Radiation Density in an Isotropic Homogeneous Universe, Mon. Not. R. Astron. Soc. 131 (1965) 1 
[59] P. S. Wesson, Olbers' Paradox and the Spectral Intensity of the Extragalactic Background Light, Astrophys. J. 367 (1991) 399

[60] S. Chandrasekar, An Introduction to the Study of Stellar Structure, Univ. Chicago Press (1939)

[61] A. C. Phillips, The Physics of Stars, Wiley (1994)

[62] F. C. Adams, Stars in Other Universes: Stellar structure with different fundamental constants, J. Cosmol. Astropart. Phys. 08 (2008) 010

[63] A. S. Burrows and J. P. Ostriker, Astronomical Reach of Fundamental Physics, Proc. Nat. Acad. Sci. 111 (2014) 2409

[64] J. D. Barrow, Varying Constants, Roy. Soc. London Trans. A 363 (2005) 2139

[65] J.-P. Uzan, The Fundamental Constants and their Variation: Observational and theoretical status, Rev. Mod. Phys. 75 (2003) 403

[66] R. Heller and J. Armstrong, Superhabitable Worlds, Astrobiology 14 (2014) 50 